\title{
Fermentation Optimization for Actinomycetes as a Single Cell Protein: A Comprehensive Review
}

Hayat Ullah ${ }^{1 *}$, Asad Ullah ${ }^{2}$, and Muhammad Waseem Khan ${ }^{3}$

${ }^{1}$ Department of Bioinformatics \& Biotechnology, Faculty of Life Science, Government College University Faisalabad-Pakistan.

${ }^{2}$ Department of Microbiology, Faculty of Life Science and Informatics, Balochistan University of Information Technology Engineering and Management Sciences, Quetta, Pakistan.

${ }^{3}$ Department of Biotechnology, Faculty of Life Sciences and Informatics, Balochistan University of Information Technology Engineering and Management Sciences, Quetta, Pakistan.

Corresponding author:*

Hayat Ullah

Department of Bioinformatics \& Biotechnology, Faculty of Life Science, Government College University Faisalabad-Pakistan, 38000

E-mail: hayatullah22@gcuf.edu.pk

Phone: +92-315-5655677

\begin{abstract}
Single cell proteins are the dead dried cells of microorganisms or purified protein isolated from microorganism's cell culture, used as a food supplement to humans' food and animals feed. World suffer from malnutrition particularly developing countries, due to rapid increase in population, increased the demand for protein and nutrients requirement. Bacteria is potential microorganism for SCP production due to high protein content, fast generation time, bio-active secondary metabolites production and can grow on various substrates. Actinobacteria species and strain have capability to produce biological active compounds, produced about two-thirds of antibiotics available in the market, actively used as antibiotics, antiprotozoal, antifungal, antiviral, anticancer, anticholesterol, antihelminth and immunosuppressant. Actinomycetes can be used as probiotic as well as single cell protein that will deal with antibiotic as well as protein source.
\end{abstract}

\section{Keywards}

Single cell protein; Probiotic; Actinomycetes; Fermentation optimization 


\section{Introduction}

The demand for necessary food increased due to rapid increase world population. Particularly protein source increasing deficiency become a serious problem for human beings. Efforts have been made for production of unconventional and alternate to proteins source, since 1996, new sources from microorganisms (yeast, bacteria, algae and fungi) were used as protein biomass [1]. In 1968 at Massachusetts Institute of Technology (SCP) 'single cell protein' term was used instead of petroprotein and microbial protein. On that time some people dislike the use of microorganism in food additives, despite that SCP idea was innovative to meet global food problem [2]. World suffer from malnutrition particularly developing countries, due to rapid increase in population, increased the demand for protein and nutrients requirement. Malnutrition is a serious problem for developing countries if not tackle proper way, can courses major crises. Therefore the production of single cell proteins are promising way to tackle protein deficiency problem worldwide [3].

Single cell proteins are the dead dried cells of microorganisms or purified protein isolated from microorganisms cell culture, used as a food supplement to humans food as vitamin carriers, aroma carriers, emulsifying aids and in animals feed as fattening poultry, calves, pigs, and fish, and also have applications in leather and paper industry [2]. SCP have high protein content (60-80\% as dry cell weight), also contains carbohydrates, lipids, vitamins, nucleic acids and minerals. It have high quantity of essential amino acid like methionine and lysine. These microbes have capability to utilize waste and cheap feedstock to produce biomass, rich in protein and amino acids. Conventional substrate (starch, fruit, molasses and fruit waste) and unconventional substrate (petroleum by products, ethanol, natural gas, lignocellulosic biomass and methanol) have been used for single cell proteins production [1]. Interest in the production of proteins from microbes for animal fodder depends on production cost in comparison to the market price competitors, like fishmeal and soya protein [4].

Probiotics are a live microorganism feed supplement which have capability to improve host intestinal micro-biota. Since last four decades probiotics have been investigated as an alternative to antibiotics growth promoter [5]. Probiotics have many important functions in poultry industry such as stability of normal microflora by competitive antagonism and exclusion, maintains metabolism by increasing production of digestive enzymes and ammonia, activates immune system and improves feed ingestion and digestion[6]. In some countries the use of antibiotics 
abolished to treat and prevent animal disease and for growth promote in livestock's due to antibiotic resistant pathogens related with animal and human disease. In Korea battery farming of chickens become causes many communicable disease such as diarrhea and constitutes a considerable economic problem for poultry growers. Probiotics survival in stomach acid depends upon strains of bacteria [7].

Nowadays most popular probiotics in the market are medications consist on different microbial strains or as feed additives. Modern approach for development of feed additives and probiotics based on applications of different microbes with different combination and production related to their prolonged storage $[6,8]$. The medications should sustain their characteristics during processing to feed and additives. For growth stimulation and maturing of livestock to enhance non-specific immunity probiotic, prebiotics ferment are used [5].

\section{Role of Actinomycetes Bio-active secondary metabolites}

Actinomycetes are Gram positive bacteria, filamentous structure which are form through asexual spores and branching filaments. Actinomycetes produce about $70 \%$ of available secondary metabolites from more than 22,000 known secondary metabolites [9]. These bacteria are widely distributed in soil and aquatic environment, consists $50 \%$ uncultivable soil microorganisms that is why it become most dominant and important group of soil microbes. Actinomycetes produce secondary metabolites includes antibiotics, enzymes, nutritional materials, cosmetics, antitumor, immune modulators, enzyme inhibitors [10].

Currently 576 species has been recognized and placed in genus Streptomyces and the number is growing every year. This number of species in genus Streptomyces make it the largest group of phylum Actinobacteria [11]. Streptomyces genus contains Gram positive bacteria, having GC rich (70\%) genetic material [10], versatile soil bacteria, having high biotransformation process and metabolite production. Their enzymes have degrading capability i.e. chitin and lignocellulose [12]. These bacteria mostly colonize in the soil and this ability is greatly facilitated by its vegetative hyphae growth that later on differentiates in to spores thus assist in further spreading deep into the environment. Streptomyces spores are semi-dormant that can survive for long period in the soil. When spore finds favorable condition like temperature, nutrients and moisture, the germ tube is generated that later on develops in to hyphae. The aerial hyphae follow a stage that set to initiates the organization of various process such as growth and cell cycle [13]. Streptomyces are known to 
make diverse compounds including enzymes and secondary metabolites. The well-known enzymes produced by Streptomyces include xylane, cellulases, proteases, lipase and used in commercial industry. The enzyme produced by Streptomyces are involved in biodegradation of different compounds in the soil thus promotes biological recycling [14].

Streptomyces have complex developmental stages such as sporulation and programed cell death (PCD). During solid sporulation culture, the development of development of compartmentalized mycelium (MI) initiates, and their compartments are established by membrane that have no cell wall. Some MI cells undergo programed cell death while most cells differentiate in to multinucleated mycelium with sporadic septa (MII) that express rodlin and chaplin protein gradually and assemble into rodlet layer which provides surface hydrophobicity for aerial mycelium to grow in the air. In Streptomyces MI fulfill the vegetative role and MII play role in reproductive stage and produce secondary metabolites[15].

\section{Streptomyces coelicolor}

Streptomyces coelicolor produce several antibiotics compounds, it have largest bacterial genome, complex life cycle including mycelial differentiation of substrate to aerial mycelium and spores formation. S. coelicolor produce various secondary metabolites and antibiotics especially prodiginines and colored actinorhodins [16]. Fermentation technology mostly used for large scale production of different economical compounds. Different microbes have been used for production of secondary metabolites but very low quantity in order to meet the market demand. Before production on large scale medium optimization is one of most critical phenomenon. Streptomyces strain differentiation during process in reactor mostly do not sporulate and their optimization mainly been focused on analysis of bio-physical parameters which include pellets clumps (mycelial grouping), media composition, $\mathrm{pH}$, oxygenation, temperature, agitation and production of secondary metabolites[15].

Enzymes are biological catalysts that reduce the catalytic activity in various biochemical pathways. Most of the enzymes are generally recognized as safe for food and feed. Industrial scale production of different enzymes was started in mid sixteen century. More enzymes are extracted from bacterial culture source. These microorganisms produce extracellular enzymes which are used in the process of decomposing and degradation of different biological material at industry level. These enzymes 
have applications in pharmaceuticals, textile industry and agriculture. In poultry industry enzymes are used to increase digestibility of feed [17].

\section{Fermentation Optimization for Streptomyces Ceolicolor}

Fermentation optimization is required to maximize product yield and reduce product cost. Medium optimization is one of the most critical investigated approach that carried out earlier before 1970s, classical methods were used to optimize medium for fermentation purpose while with new technology modern mathematical techniques used, that results media optimization become more effective, vibrant, efficient. Medium optimization includes physical parameters such as $\mathrm{pH}$, temperature, agitation, aerations and medium components such as carbon, nitrogen and phosphate must be identified and optimized accordingly. The use of mathematical method for media optimization can overcome the limitations of classical OFAT method and can be more efficient tool for optimization of secondary metabolite production. Changing more than one component at a time in the medium can be more effective than changing only one component at a time $[18,19]$.

\section{$\underline{\text { Media Composition }}$}

Carbon, nitrogen and phosphates are basic medium components. Microbes use carbon used as energy source and have importance for their growth and metabolites production. Galactose used by marine bacteria enhance their secondary metabolites production. Medium components covers significant portion of product cost and the rate of assimilation of carbon source also affects product [20].

Nitrogen source and its concentration in medium are as important as carbon. Microbes have capability to utilize both nitrogen source (organic or inorganic). In some cases the use of particular amino acid may increase its productivity while unsuitable amino acids can decrease the production of secondary metabolites [21].

Phosphate is another important medium component required for production phospholipids and nucleic acids. The amount of nitrogen required for fermentation medium depends upon composition of broth and need of microbe. Mostly phosphate low concentration required for secondary metabolite production initiation while in some cases it suppress primary and secondary metabolite production [22]. 


\section{Temperature and $\mathrm{pH}$}

$\mathrm{pH}$ and temperature optimization is important for antibiotics production as it effects bacterial cell growth, secondary metabolites production [23]. Streptomyces produce optimum secondary metabolites at $35 \mathrm{C}$ and $7.0 \mathrm{pH}$ while extreme temperature and $\mathrm{pH}$ unfavorable for secondary metabolite production [24]. Temperature is more important factor that in all fermentation due to its impact on microorganism growth and secondary metabolite production. The optimum incubation temperature is $32 \mathrm{C}$. The temperature exerts same effect on growth of microbe and production of enzyme irrespective of the mode of fermentation [25].

\section{Oxygen Transfer}

Oxygensparingly soluble in culture medium and in liquid ensuring availability of sufficient oxygen transfer appears critical. The supply of oxygen to liquid phase and access to growing microbial cells can raise technical issues related to productivity of microbes. Dissolve oxygen tension (DOT), strongly effects on the production of secondary metabolites such as concentration of antibiotics, enzymes activity, and induction of antibiotics production and morphology of Streptomyces. For Streptomyces species broths may indicates a non-Newtonian rheology which increase complexity of oxygen mass transfer. To enhance oxygen transfer more mixing is often chosen [26]. The terms used for the quantification of oxygen transfer are oxygen uptake rate (OUR) or oxygen transfer rate (OTR), and gas-liquid mass transfer coefficient. Oxygen is transferred to liquid phase from rising gas bubble during aerobic bioprocess which is decomposed into three steps such as gas phase, liquid phase and microbial phase. During first phase oxygen is transferred from inside bubble to gas film than gas transferred to gas liquid interface and to bulk liquid and to the liquid around the cell. Around bubbles, the liquid film resistance usually control the rate of transfer outside microbial pellet [27].

\section{$\underline{\text { Agitation effects }}$}

Agitation effects the production of many secondary metabolites by effecting growth rate, cell damage, product synthesis, metabolism and enzyme activity, DOT, broth viscosity, and morphology of species. An optimal agitation exist but in aerobic submerged fermentation physiological response to mixing effects remain ambiguous. Homogeneity in stirred and sparged bioreactors the expected rate of turbulent dissipation reach 100 times and the determination of gas 
dissipation rates liquid reactors raises key problems such as the used if laser technique in bubbly flows [28]. Increase in agitation rates promotes higher Streptomyces biomass concentration, however in shaking flask simultaneous increase in oxygen transfer could seriously be consider for increase in biomass. Increase in agitation intensity also influence substrate consumption with modification in morphology of Streptomyces species and also modulate the production of secondary metabolites [27].

\section{Acknowledgments}

No acknowledgments

\section{Conflict of Interest}

The authors declare that they have no conflict of interest.

\section{REFERENCES}

1. Suman, G., et al., Single cell protein production: a review. 2015. 4(9): p. 251-262.

2. Srividya, A., et al., International Journal for Pharmaceutical Research Scholars (IJPRS).

3. Ali, S., et al., Production and Processing of Single Cell Protein (SCP)-A Review. 2017. 4(7): p. 86-94.

4. Parajó, J., et al., NH 4 OH-Based pretreatment for improving the nutritional quality of single-cell protein (SCP). 1995. 55(2): p. 133-149.

5. Jiang, T., et al., Oral delivery of probiotics in poultry using pH-sensitive tablets. 2017. 27(4): p. 739-746.

6. Ajuwon, K.J.J.o.A.P.R., Toward a better understanding of mechanisms of probiotics and prebiotics action in poultry species. 2015. 25(2): p. 277-283.

7. Koshchaev, A.G., et al., Screening of microorganism symbiont strains as a base of probiotics for poultry industry. 2017. 9(8): p. 1373.

8. Sugiharto, S., et al., Dietary supplementation of probiotics in poultry exposed to heat stress-a review. 2017. 17(3): p. 591-604.

9. Uyeda, M.J.A., Fattiviracins, antiviral antibiotics produced by an actinomycete. 2003. 17(2): p. 57-66.

10. Rotich, M.C., Bio-Prospecting for Broad Spectrum Antibiotic Producing Actinomycetes Isolated from Virgin Soils in Kericho County. 2018, JKUAT-COHES.

11. Kieser, T., et al., Practical Streptomyces Genetics (The John Innes Foundation, Norwich, $U K, 2000) .2004$. 
12. de Lima Procópio, R.E., et al., Antibiotics produced by Streptomyces. 2012. 16(5): p. 466471.

13. Arul Jose, P., et al., Characterization of antibiotic producing rare actinomycete Nonomuraea sp. JAJ18 derived from an Indian coastal solar saltern. 2014. 2014.

14. McCarthy, A.J. and S.T.J.G. Williams, Actinomycetes as agents of biodegradation in the environment-a review. 1992. 115(1): p. 189-192.

15. Rioseras, B., et al., Mycelium differentiation and development of Streptomyces coelicolor in lab-scale bioreactors: programmed cell death, differentiation, and lysis are closely linked to undecylprodigiosin and actinorhodin production. 2014. 151: p. 191-198.

16. Wentzel, A., et al., Optimized submerged batch fermentation strategy for systems scale studies of metabolic switching in Streptomyces coelicolor A3 (2). 2012. 6(1): p. 59.

17. Dodd, D. and I.K.J.G.B. Cann, Enzymatic deconstruction of xylan for biofuel production. 2009. 1(1): p. 2-17.

18. Elibol, M., Optimization of medium composition for actinorhodin production by Streptomyces coelicolor A3 (2) with response surface methodology. Process Biochemistry, 2004. 39(9): p. 1057-1062.

19. Wentzel, A., et al., Optimized submerged batch fermentation strategy for systems scale studies of metabolic switching in Streptomyces coelicolor A3 (2). BMC systems biology, 2012. 6(1): p. 59.

20. Elibol, M., Response surface methodological approach for inclusion of perfluorocarbon in actinorhodin fermentation medium. Process Biochemistry, 2002. 38(5): p. 667-673.

21. Rioseras, B., et al., Mycelium differentiation and development of Streptomyces coelicolor in lab-scale bioreactors: programmed cell death, differentiation, and lysis are closely linked to undecylprodigiosin and actinorhodin production. Bioresource technology, 2014. 151: p. 191-198.

22. Niladevi, K.N., et al., Optimization of laccase production from a novel strainStreptomyces psammoticus using response surface methodology. Microbiological research, 2009. 164(1): p. 105-113.

23. Rajnisz, A., et al., Characterization and optimization of biosynthesis of bioactive secondary metabolites produced by Streptomyces sp. 8812. 2016. 65(1): p. 51-61. 
24. Narayana, K. and M.J.R.J.P. Vijayalakshmi, Optimization of antimicrobial metabolites production by Streptomyces albidoflavus. 2008. 2(1): p. 4-7.

25. Niladevi, K.N., et al., Utilization of rice straw for laccase production by Streptomyces psammoticus in solid-state fermentation. 2007. 34(10): p. 665-674.

26. Petersen, N., et al., In situ near infrared spectroscopy for analyte-specific monitoring of glucose and ammonium in streptomyces coelicolor fermentations. Biotechnology progress, 2010. 26(1): p. 263-271.

27. Olmos, E., et al., Effects of bioreactor hydrodynamics on the physiology of Streptomyces. 2013. 36(3): p. 259-272.

28. Comba, S., et al., Engineering a Streptomyces coelicolor biosynthesis pathway into Escherichia coli for high yield triglyceride production. Biotechnology for biofuels, 2014. 7(1): p. 172. 